\title{
AN OPTIMUM PROBLEM IN THE WEINSTEIN METHOD FOR EIGENVALUES
}

\author{
H. F. WEINBERGER
}

1. Introduction. The method of Weinstein [1] gives upper bounds for the eigenvalues $\lambda_{1}^{\prime} \geq \lambda_{2}^{\prime} \geq \cdots$ of the projection $L^{\prime}$ into a space \& of a completely continuous positive symmetric operator $L$ in a Hilbert space $\sqrt{2}$ with eigenvalues $\lambda_{1} \geq \lambda_{2} \geq \ldots$. These upper bounds are the eigenvalues $\lambda_{n}^{(m)}$ of the projection of $L$ into a space of finite index $m$,

$$
\mathcal{F}_{2} \ominus\left\{p_{1}, \cdots, p_{m}\right\}
$$

where $p_{1}, \cdots, p_{m}$ are any vectors in the space

$$
\mathfrak{B}=\mathscr{S} \Theta B \text {. }
$$

The chief part of the Weinstein method is the explicit determination of the eigenvalues $\lambda_{n}^{(m)}$ in the space (1) in terms of the eigenvalues and eigenvectors of $L$ in $\mathscr{F}_{2}$. These satisfy

$$
\lambda_{n}^{(m)} \geq \lambda_{n}^{\prime}
$$

The values $\lambda_{n}^{(m)}$ will, of course, depend on the choice of the vectors $\left(p_{1}\right.$, $\left.\ldots, p_{m}\right)$. It is naturally desirable that the upper bound for a particular eigenvalue $\lambda_{n}^{\prime}$ should be as small as possible. This paper investigates how small it can be made for given $n$ and $m$ by a proper choice of the constraint vectors $\left(p_{1}, \cdots, p_{m}\right)$.

Because of the minimax principle, $\lambda_{n}^{(m)}$ must satisfy

$$
\lambda_{n}^{(m)} \geq \lambda_{n+m}
$$

Our result is that the inequalities (3) and (4) are the only restrictions on the smallness of $\lambda_{n}^{(m)}$. In other words, for given $n$ and $m$, there exist vectors $\left(p_{1}\right.$, $\cdots, p_{m}$ ) such that the weaker of the inequalities (3) and (4) becomes an equality.

Received December 12, 1951. This work was sponsored by the Office of Naval Research.

Pacific J. Math. 2 (1952), 413-418 
2. The case of a single constraint. We first prove our result for the case of the first intermediate problem, that is, for $m=1$.

THEOREM 1. For any given $n$, there is a vector $p$ in the space

$$
\Re=\mathscr{L} \Theta \Leftrightarrow
$$

such that, if the projection of $L$ into $\mathcal{F}_{\mathcal{C}} \Theta\{p\}$ has eigenvalues

$$
\lambda_{1}^{(1)} \geq \lambda_{2}^{(1)} \geq \cdots
$$

either

$$
\lambda_{n}^{(1)}=\lambda_{n}^{\prime}
$$

or

$$
\lambda_{n}^{(1)}=\lambda_{n+1}
$$

according as $\lambda_{n}^{\prime}$ or $\lambda_{n+1}$ is larger.

Proof. If $\lambda_{n}^{\prime}=\lambda_{n}$, then (6) is satisfied for any $p$ and there is nothing to prove. Our theorem thus naturally splits into the two cases $\lambda_{n}>\lambda_{n}^{\prime} \geq \lambda_{n+1}$ and $\lambda_{n}^{\prime}<\lambda_{n+1}$, which we shall prove separately.

3. The case $\lambda_{n}>\lambda_{n}^{\prime} \geq \lambda_{n+1}$. Let the eigenvector of $L^{\prime}$ corresponding to $\lambda_{n}^{\prime}$ be $u_{n}^{\prime}$. Its eigenvalue equation can be written in terms of the operator $L$ as

$$
L u_{n}^{\prime}-\lambda_{n}^{\prime} u_{n}^{\prime}=p
$$

where $p$ is some vector in $\Re$. Let us assume for the moment that $p$ is not a null vector. Then (8) is an eigenvalue equation for the projection of $L$ into $\&$ but not for $L$. Any eigenvector of $L$ corresponding to the eigenvalue $\lambda_{n}^{\prime}$ must, because of (8), be orthogonal to $p$ and hence must belong to $\sqrt{2} \Theta\{p\}$. Thus, the multiplicity of $\lambda_{n}^{\prime}$ as an eigenvalue of the projection of $L$ into $\mathscr{L}_{2} \Theta\{p\}$ is one greater than its multiplicity as an eigenvalue of $L$. Let the latter be $r \geq 0$. If $r=0$, then $\lambda_{n}^{\prime}>\lambda_{n+1}$, and $\lambda_{n}^{\prime}$ must be $\lambda_{n}^{(1)}$ by the minimax principle. If $r \geq 1$, then $\lambda_{n}>\lambda_{n+1}=\cdots=\lambda_{n+r}>\lambda_{n+r+1}$, and the minimax principle gives

$$
\lambda_{n-1}^{(1)} \geq \lambda_{n}>\lambda_{n}^{\prime} \geq \lambda_{n+1}>\lambda_{n+r+1} \geq \lambda_{n+r+1}^{(1)}
$$

Thus, since the multiplicity of $\lambda_{n}^{\prime}$ in $\mathscr{F} \Theta\{p\}$ is $r+1$, we must have 


$$
\lambda_{n}^{(1)}=\lambda_{n}^{\prime}
$$

so that the vector $p$ in (8) has the property stated in our theorem.

If $\lambda_{n}^{\prime}=\lambda_{n+1}$, it is possible that the vector $p$ in (8) is a null vector. This means that the eigenvector of $L^{\prime}$ corresponding to $\lambda_{n}^{\prime}$ is also an eigenvector of $L$. Suppose that the same is also true of the eigenvalues $\lambda_{n+1}^{\prime}, \cdots, \lambda_{n+s-1}^{\prime}$ but not of $\lambda_{n+s}^{\prime}$. We then consider the projections $\bar{L}$ and $\bar{L}^{\prime}$ into

$$
\mathcal{F}_{2} \ominus\left\{u_{n}^{\prime}, \cdots, u_{n+s-1}^{\prime}\right\} \text { and } \circledast \Theta\left\{u_{n}^{\prime}, \cdots, u_{n+s-1}^{\prime}\right\}
$$

respectively, and call their eigenvalues $\bar{\lambda}_{i}$ and $\bar{\lambda}_{i}^{\prime}$. Then $\bar{L}^{\prime}$ has the same eigenvalues as $L^{\prime}$, except that the eigenvalues $\lambda_{n}^{\prime}, \cdots, \lambda_{n+s-1}^{\prime}$ are removed. The same is true of $\bar{L}$ and $L$. Then

$$
\overline{\lambda_{n}^{\prime}}=\lambda_{n+s}^{\prime} \leq \lambda_{n}^{\prime}
$$

If there is a vector $p$ in $\Re$ so that the $n$-th eigenvalue of $L$ in

$$
\mathscr{L} \ominus\left\{u_{n}^{\prime}, \cdots, u_{n+s-1}^{\prime}, p\right\}
$$

is at most $\bar{\lambda}_{n}^{\prime}$, then, because of (11), the $n$-th eigenvalue of $L$ in $\mathscr{F} \Theta\{p\}$ is $\lambda_{n}^{\prime}$ and equation (6) in our theorem will be proved. Now if

$$
\bar{\lambda}_{n}^{\prime}=\lambda_{n+s}^{\prime} \geq \bar{\lambda}_{n},
$$

then, since by definition of $s$ the eigenvector of $L^{\prime}$ corresponding to $\lambda_{n+s}^{\prime}$ is not an eigenvector of $L$, the existence of such a vector $p$ follows from the first part of this paragraph. If, on the other hand, we have

$$
\lambda_{n}^{\prime}<\bar{\lambda}_{n+1},
$$

the existence of this vector $p$ will be assured by the results of the next paragraph.

A final possibility* is that there is no integer $s$ such that the eigenvector $u_{n+s}$ of $L^{\prime}$ is not also an eigenvector of $L$. In other words, all but the first $n-1$ eigenvectors of $L^{\prime}$ are also eigenvectors of $L$. Then, since $\lambda_{n}^{\prime}=\lambda_{n+1}$, the vectors $u_{1}^{\prime}, \cdots, u_{n-1}^{\prime}$ are the only eigenvectors of $L^{\prime}$ which are not orthogonal to $u_{1}, u_{2}, \cdots, u_{n}$. Therefore there is one linear combination $p$ of $u_{2}, \cdots, u_{n}$ which is orthogonal to all eigenvectors of $L^{\prime}$ and hence belongs to $\mathfrak{P}$. There can

${ }^{*}$ This possibility was pointed out by C. Arf in the course of an alternative proof of the results here presented. 
be at most $n-1$ eigenvectors of the projection of $L$ into $\mathscr{K} \Theta\{p\}$ which are not orthogonal to $u_{1}, \cdots, u_{n}$. Therefore, the $n$-th eigenvalue of this projection is $\lambda_{n+1}=\lambda_{n}^{\prime}$, and both equalities (6) and (7) hold.

4. The case $\lambda_{n}^{\prime}<\lambda_{n+1}$. We now show that if $\lambda_{n}^{\prime}<\lambda_{n+1}$ then the equation (7) can be made to hold. This will be done by induction. We first replace the space $\Re$ by a finite space. Since $L$ is completely continuous it follows that $\lambda_{m} \rightarrow 0$ as $m \rightarrow \infty$; therefore there is an integer $m$ such that

$$
\lambda_{n+1} \geq \lambda_{n}^{\prime}+\lambda_{m+1}
$$

It has been shown by the author $[1,2]$ that if we let $p_{i}$ be the projection in $\Re \beta$ of $u_{i}$, then the eigenvalues $\lambda_{n}^{(m)}$ of $L$ in $\mathcal{F}_{2} \Theta\left\{p_{1}, \cdots, p_{m}\right\}$ satisfy

$$
\lambda_{n}^{(m)} \leq \lambda_{n}^{\prime}+\lambda_{m+1}
$$

Combining this with (14), we obtain

$$
\lambda_{n}^{(m)} \leq \lambda_{n+1}
$$

Thus, it will suffice to show that if the inequality (16) holds where $\lambda_{n}^{(m)}$ is the $n$-th eigenvalue of $L$ in a space $\mathscr{F}_{\mathcal{C}} \Theta\left\{p_{1}, \ldots, p_{m}\right\}$, then there is a linear combination $p$ of the vectors $p_{1}, \cdots, p_{m}$ such that the $n$-th eigenvalue of $L$ in $\mathscr{S}_{2} \Theta\{p\}$ is $\lambda_{n+1}$. Our induction proof consists of showing that if (16) holds for $m>1$ then there is a linear combination $p^{\prime}$ of $p_{m-1}$ and $p_{m}$ such that the $n$-th eigenvalue of $L$ in $\mathcal{F}_{2} \Theta\left\{p_{1}, \ldots, p_{m-2}, p^{\prime}\right\}$ is at most $\lambda_{n+1}$. If $\lambda_{n}^{(m-1)} \leq \lambda_{n+1}$, this is obviously true, for we must only take $p^{\prime}=p_{m-1}$. Thus, we need to examine only the case

$$
\lambda_{n}^{(m-2)} \geq \lambda_{n}^{(m-1)}>\lambda_{n+1} \geq \lambda_{n}^{(m)}
$$

Since, by the minimax theorem,

$$
\lambda_{n+1} \geq \lambda_{n+1}^{(m-2)}
$$

our induction step will be proved if we find $p$ so that the $n$-th eigenvalue of $L$ in $\mathcal{F}_{2} \Theta\left\{p_{1}, \cdots, p_{m-2}, p^{\prime}\right\}$ is equal to either $\lambda_{n}^{(m)}$ or $\lambda_{n+1}^{(m-2)}$. In other words, the induction step is just Theorem 1 in the special case in which $\beta$ is a 2space.

Thus if $\lambda_{n}^{(m)} \geq \lambda_{n+1}^{(m-2)}$ the induction is proved by the results of $\S 3$. Note that in the case of a common eigenvector where one had to reduce the proof 
in $\S 3$ to the proof of this section, the reduction is to the case $\lambda_{n}^{(m)}<\lambda_{n+1}^{(m-2)}$, which will now be treated.

If $\lambda_{n}^{(m)}<\lambda_{n+1}^{(m-2)}$, we must construct a linear combination $p^{\prime}$ of $p_{m-1}$ and $p_{m}$ so that $\lambda_{n+1}^{(m-2)}$ is the $n$-th eigenvalue of $L$ in $\mathcal{F}_{2} \Theta\left\{p_{1}, \cdots, p_{m-2}, p^{\prime}\right\}$. To do this, we take for $p^{\prime}$ the linear combination of $p_{m-1}$ and $p_{m}$ which is orthogonal to the eigenvector corresponding to $\lambda_{n+1}^{(m-2)}$. Then $\lambda_{n+1}^{(m-2)}$ is an eigenvalue of $L$ in $\mathscr{S C}_{\mathcal{C}} \Theta\left\{p_{1}, \cdots, p_{m-2}, p^{\prime}\right\}$. By the minimax principle, the $(n+1)$-st eigenvalue in this space is at most $\lambda_{n}^{(m)}<\lambda_{n+1}^{(m-2)}$. Therefore $\lambda_{n+1}^{(m-2)}$ must be the $n$-th eigenvalue in this space, and $p^{\prime}$ has the desired property.

Thus, our induction step is proved and Theorem 1 has been shown to hold in all possible cases.

5. The general intermediate problem. We are now in a position to prove the more general result announced in the introduction.

THEOREM 2. For any fixed integers $m$ and $n$, there are vectors $p_{1}, \cdots, p_{m}$ in $\mathfrak{s}$ which, if used as constraints in the n-th intermediate problem, yield either

$$
\lambda_{n}^{(m)}=\lambda_{n}^{\prime}
$$

or

$$
\lambda_{n}^{(m)}=\lambda_{n+m}
$$

Proof. We first prove the possibility of the equality (20) when

$$
\lambda_{n+m}>\lambda_{n}^{\prime}
$$

According to Theorem 1 with $n+m-1$ substituted for $n$, there is a vector $p_{1}$ such that

$$
\lambda_{n+m-1}^{(1)}=\lambda_{n+m}
$$

We then apply Theorem 1 to the projection of $L$ into $\mathcal{F}_{2} \Theta\left\{p_{1}\right\}$ to assert the existence of a vector $p_{2}$ such that

$$
\lambda_{n+m-2}^{(2)}=\lambda_{n+m}
$$

This process is repeated until the equality (20) is obtained. Inequality (21) assures us that the equality (7) of Theorem 1 will always be attainable.

If $\lambda_{n+m} \leq \lambda_{n}^{\prime}$, then there is an integer $l \leq m$ such that 


$$
\lambda_{n+l} \leq \lambda_{n}^{\prime}<\lambda_{n+l-1}
$$

We shall show that there are $l$ vectors $p_{1}, \cdots, p_{l}$ for which

$$
\lambda_{n}^{(l)}=\lambda_{n}^{\prime}
$$

The equality (19) will then hold for any $m-1$ vectors $p_{l+1}, \cdots, p_{m}$ appended to the first $l$.

Since $\lambda_{n}^{\prime} \geq \cdots \geq \lambda_{n+l-1}^{\prime}$, we can proceed as in the proof of (20) to show that there are $l-1$ vectors $p_{1}, \cdots, p_{l-1}$ for which

$$
\lambda_{n+1}^{(l+1)}=\lambda_{n+l}
$$

We now apply Theorem 1 to $L$ in $\mathcal{F}_{\mathcal{C}} \Theta\left\{p_{1}, \cdots, p_{l-1}\right\}$. According to (24) and (26) it is the equality (6) which can be made to hold by a constraint $p_{l}$. We thus obtain (25), and Theorem 2 is proved.

\section{REFERENCES}

1. H. F. Weinberger, An error estimate for the method of Weinstein. Abstract of a paper presented at the Minneapolis meeting of the American Mathematical Society, 1951.

2. ——, An error estimate for the Weinstein method for eigenvalues. Submitted to Proc. Amer. Math. Soc.

3. A. Weinstein, Etude des spectres des équations aux dérivées partielles de la théorie des plaques elastiques, Memorial des Sciences Mathematiques, 88, GauthierVillars, Paris, 1937.

4. N. Aronszajn, Rayleigh-Ritz and A. Weinstein methods for approximation of eigenvalues. Proc. Nat. Acad. Sci. 34 (1948) 474- 480 and 594-601.

5. N. Aronszajn and A. Weinstein, On the unified theory of eigenvalues of plates and membranes. Amer. J. Math. 64 (1942) 623-645.

Institute for Fluid Dynamics and Applied Mathematics

UNIVERSITY OF MARYLAND 